\title{
Multi-Ensayos
}

Vol. 8, núm. 15

ISSN: 2412-3285

https://multiensayos.unan.edu.ni

DOI: https://doi.org/10.5377/multiensayos.v8i15.13189

\section{El nuevo enfoque de la extensión universitaria como instrumento de vinculación hacia sectores vulnerables en la Facultad de Ciencias Económicas de la Universidad Nacional de Asunción. Año 2020}

\section{The new approach to university extension as an instrument of linkage towards vulnerable sectors in the Faculty of Economic Sciences of the National University of Asunción. Year 2020}

\author{
Celina González de Álvarez \\ Ana Leticia Carosini Ruiz Diaz²
}

Recibido: 15 de julio de 2021. Aceptado: 12 de noviembre de 2021

\section{RESUMEN}

El presente artículo esboza el papel preponderante que la Academia puede cumplir durante esta Pandemia con la articulación de las actividades de extensión universitaria a fin de que los estudiantes puedan aplicar los conocimientos adquiridos en situaciones de la realidad circundante, especialmente hacia sectores vulnerables de la comunidad. Es por ello que se aborda en este artículo el nuevo enfoque dado a las actividades de extensión universitaria como herramienta fundamental para que la educación superior asista a las necesidades de la población y se posicione en la mente de los ciudadanos como una institución no solo formadora de profesionales, sino que además se convierta en un laboratorio concreto de acciones positivas para la sociedad. Se analizaron para ello las principales actividades que la Facultad de Ciencias Económicas de la Universidad Nacional de Asunción ha implementado, a través de la extensión universitaria, evidenciando la necesidad de plantear el procedimiento efectivo para garantizar que los programas desarrollados con sectores vulnerables desde el año 2017 no se viesen suspendidos en el año 2020 de la Pandemia de la COVID19; lo cual fue logrado con la adopción de un cambio de enfoque de la extensión universitaria efectivamente.

Palabras clave: extensión universitaria; vinculación; sectores vulnerables.

1 Docente Técnica en el área de Extensión Universitaria. Dirección de Extensión y Servicios - FCE UNA; cgonzalez283@eco.una.py 2 Docente Técnica en el área de Investigación. Dirección de Investigación FCE-UNA; alcarosini@eco.una.py (c) 2022 Revista Multi-Ensayos. 


\section{ABSTRACT}

This article outlines the preponderant role that the Academy can fulfill during this Pandemic with the articulation of university extension activities so that students can apply the knowledge acquired in situations of the surrounding reality, especially towards vulnerable sectors of the community. That is why this article addresses the new approach given to university extension activities as a fundamental tool for higher education to attend to the needs of the population and to position itself in the minds of citizens as an institution that is not only a professional training institution, but also become a concrete laboratory of positive actions for society. For this, the main activities that the Faculty of Economic Sciences of the National University of Asunción has implemented, through university extension, were analyzed, evidencing the need to propose an effective procedure to guarantee that the programs developed with vulnerable sectors since 2017 they were not suspended in the year 2020 of the COVID19 Pandemic; which was achieved with the adoption of a change of focus of the university extension effectively.

Keywords: university extension; linking; vulnerable sectors.

\section{INTRODUCCIÓN}

Paraguay cuenta con la Ley de Educación Superior $N^{\circ} 4.995 / 2013$, que define en el artículo $22^{\circ}$ a las universidades como las instituciones de educación superior IES que abarcan una multiplicidad de áreas específicas del saber en el cumplimiento de su misión de investigación, enseñanza, formación y capacitación profesional, extensión y servicio a la comunidad. Además, se establece en el artículo $N^{\circ} 24$ los fines que deben tener las universidades explícitamente en: a) El desarrollo de la personalidad humana inspirada en los valores de la ética, de la democracia y la libertad; b) La enseñanza y la formación profesional; c) La investigación en las diferentes áreas del saber humano; d) La formación de una racionalidad reflexiva y crítica y de la imaginación creadora; e) El servicio a la colectividad en los ámbitos de su competencia; f) El fomento y la difusión de la cultura universal y en particular de la nacional; g) La extensión universitaria; y h) El estudio de la problemática nacional.

La Universidad Nacional de Asunción UNA, como primera IES que tuvo origen el 9 de julio de 1956 con la promulgación de la Ley N³56"Que establece la carta orgánica de la Universidad Nacional de Asunción"; desde su concepción, ya estableció a la extensión universitaria como uno de sus fines en virtud a que, para esa época, dicha actividad cobraba fuerza como función sustantiva de la academia.

Con el transcurrir de los años, y como producto de la capitalización de experiencias que condujeron a cumplir con la función sustantiva, la UNA elaboró el Reglamento General de Extensión Universitaria, aprobado en fecha 2 de noviembre de 2005 conforme al Acta No. 22/02/11/2005 y Resolución No. 42800-2005, en el cual fueron delineadas las actividades que se enmarcan dentro de la extensión universitaria para facilitar a los docentes y estudiantes la grilla de acciones a ejecutar.

La UNA define a la extensión Universitaria en el citado Estatuto vigente como "un proceso pedagógico transformador y de compromiso social que contribuye al desarrollo inclusivo, sostenible y sustentable de la sociedad paraguaya y su entorno regional e internacional para compartir conocimientos científicos, empíricos, tecnológicos y culturales". En este contexto, la extensión universitaria es considerada como una de las funciones esenciales que, conjuntamente con la investigación y el área académica, constituyen 
los pilares básicos sobre los que se construye un modelo de universidad democrática y comprometida socialmente, en su permanente búsqueda de la verdad camino a la excelencia.

En esa línea de acción, desde el año 2017, la Facultad de Ciencias Económicas (FCE) de la UNA, a través de la Dirección de Extensión y Servicios (DES), promueve acciones de la extensión universitaria vinculadas a las actividades económicas del país, principalmente aquellas relativas a las áreas productivas, como sector primario vinculadas al agro y la ganadería, microempresarios urbanos y rurales, emprendedores, entre otros; con el objetivo de transferir los beneficios producidos por el conocimiento económico, administrativo y contable mediante la implementación de programas de capacitación extracurriculares, asistencia social, actividades culturales y deportivas, promoviendo así entre los estudiantes el fortalecimiento de una conciencia de responsabilidad y compromiso con su entorno.

Asimismo, la FCE-UNA, a través de la DES, ha desarrollado programas de extensión universitaria, que han tenido continuidad en los años 2018 y 2019, a fin de brindar atención prioritaria a un segmento de la población, que en muchos casos no es atendido por las instancias públicas y privadas, y donde la Academia puede tener una presencia más proactiva, con el objetivo de dar cumplimiento al nuevo paradigma de la educación superior relativo a la promoción del conocimiento y a la práctica solidaria y formativa, realizando la vinculación de Universidad, Sociedad, Estado, con miras a contribuir a la mejora de la calidad de vida de la población.

Con la pandemia COVID191', el mundo entero ha estado experimentando una profunda transformación que implica reinventarse para proseguir su rumbo. Esta situación no es ajena a la Academia, y por ello, la FCE-UNA acorde al compromiso social asumido, ha iniciado el proceso de incorporar estrategias tecnológicas que permitan, más que nunca, acompañar a los sectores vulnerables y brindar asistencia técnica a los mismos, principalmente a aquellos ubicados en su área de influencia, ya sea en la Sede Central en la ciudad de San Lorenzo, como en las filiales ubicadas en los departamentos de Central, Cordillera, Guairá, Paraguarí, Caaguazú, San Pedro y Presidente Hayes en Paraguay.

Para este trabajo se adopta el concepto de vulnerabilidad aplicando a aquellos sectores o grupos de la población que por su condición de edad, sexo, estado civil y origen étnico se encuentran en condición de riesgo que les impide incorporarse al desarrollo y acceder a mejores condiciones de bienestar. Como se ha señalado, involucra no solo a comunidades que implementan actividades productivas o comerciales a nivel microempresarial, sino también a conglomerados conocidos como asentamientos urbanos, en los cuales existe precariedad tanto en lo económico como en el acceso a servicios básicos.

En el presente artículo se presenta el esfuerzo del trabajo en equipo realizado por la DES de la FCE-UNA con respecto a llevar a cabo las actividades de extensión desarrolladas con los sectores más vulnerables, en el periodo 2017 al 2019, y que en el año 2020, cuya singular característica ha sido la Pandemia, se han mantenido activas con la ayuda de las herramientas de las Tecnologías de Información y Comunicación (TIC), posibilitando que la extensión universitaria contribuya a cumplir los fines de la Academia en su vinculación con la sociedad.

1 COVID: Según la Organización Mundial de la Salud (OMS) es una enfermedad respiratoria muy contagiosa causada por el virus SARS-CoV-2. 


\section{El papel de la extensión universitaria en la sociedad}

El concepto de extensión universitaria ha recorrido un largo trayecto desde sus primeras formulaciones durante el siglo XIX. A lo largo del mismo ha ido incorporando distintos significados, generalmente enmarcados en un modelo de relación asimétrica entre la sociedad y la población asistida.

Para Di Mari (2013), el concepto de apropiación social del conocimiento, de factura más reciente, pretende una aproximación más efectiva y dinámica del público al conocimiento científico. Sin embargo, ambos conceptos mantienen de manera más o menos explícita la relación lineal y asimétrica entre el conocimiento experto y el conocimiento profano.

Para Tomassino \& Cano (2016) se viene cíclicamente debatiendo en las instituciones de educación superior la posibilidad de ubicar la extensión universitaria entre las funciones académicas cuyo origen se sitúa en la idea de que la aparición de la difusión de la cultura y la extensión universitaria, como funciones sustantivas en las propias leyes orgánicas de las universidades, lo que implica automáticamente su consideración como función académica.

Definir el carácter académico de la extensión o de cualquier otra función necesariamente requiere reconsiderar el tipo de actividades que se incluyen dentro de este concepto. Las actividades académicas se circunscriben regularmente a las labores de docencia e investigación, mismas que implican un delicado equilibrio entre libertades (de cátedra y de investigación) y exigencias de rigor metodológico, originalidad y comportamientos acordes con la ética de las disciplinas.

La educación superior y con mayor razón, la universidad pública, es preciso que contenga como componente prominente la vinculación social que denote la marcada responsabilidad social universitaria que se concreta en la actuación conjunta para contribuir al mejoramiento económico y social de su entorno comunitario.

Tal como lo menciona, Chuaqui (2002) la universidad es una de las más grandes creaciones de la civilización occidental, única en su género: un instituto dedicado al mundo del intelecto. La universidad nació no de una idea preconcebida, sino de la paulatina convergencia de circunstancias históricas, entre dos corrientes de personas: aquellas personas que querían aprender y la de los que estaban dispuestos a enseñar.

La extensión universitaria es el elemento más dinámico e integrador del vínculo universidad sociedad pues la misma, cumple una tarea crucial a través de esta función, expresada en el conjunto de acciones desplegadas dentro y fuera de sus instalaciones, mayormente de carácter educativo; principalmente cuando se relaciona con la sociedad a través de acciones concretas como cursos de capacitación sobre educación financiera, abordaje comunitario para caracterizar a poblaciones vulnerables, entre otras.

La extensión está encaminada a sus estudiantes, trabajadores y comunidad en general con la metodología de promocionar una cultura cosmopolita que refleje las necesidades, intereses y potencialidades culturales del objeto de atención, aprovechando al máximo las posibilidades científicas y educativas que 
posee la institución, así como su capacidad de identificación e interpretación del contexto social en que se desenvuelve. En este sentido, una de las actividades de extensión universitaria como las pasantías laborales posibilita la vinculación del futuro egresado con la organización empresarial que demanda mano de obra en el mercado de trabajo.

El marcado enfoque de crear un profesional comprometido con la solución de los problemas de la realidad social, consolida una mayor formación de las habilidades del futuro profesional. El estudiante desde los primeros años al tener un mayor intercambio y contacto con el medio consolida sus conocimientos y alcanza una mayor calidad en su formación profesional.

Sostiene Del Huerto (2006) que la razón de ser de la universidad contemporánea es satisfacer las necesidades sociales e individuales, a partir de la preparación del ser humano como ente activo en la sociedad en la que se desempeña.

Entonces, resulta de vital importancia que las unidades académicas que conforman una universidad, promuevan en sus estudiantes el deseo de participar como agente de su propio desarrollo. El cumplimiento de este encargo social de la universidad se concreta en la preservación, desarrollo y promoción de la cultura que en su interrelación dialéctica son la expresión de la integración de las funciones sustantivas de la universidad docencia-investigación y extensión.

Sin dudas, la vía más realista de realizar la extensión universitaria con vinculación social es a través de la participación de estudiantes y profesores en actividades de intercambio con la comunidad, con un alto nivel de pertinencia y en sectores vulnerables.

Esta vinculación recae con mayor obligatoriedad en una universidad pública ya que la misma debe fijar como uno de sus objetivos principales la retribución social a la población que la financia.

En los paradigmas actuales de la universidad, Di Mari (2013) menciona que la extensión se considera como una función totalizadora presente en cada uno de los procesos y eslabones estructurales de la educación superior, jugando un papel decisivo en la proyección social de la universidad.

La extensión universitaria en la UNA acompaña activamente las sucesivas transformaciones y cambios demográficos, económicos y socio-políticos del país, lo cual se ha plasmado en las normativas como es el caso del Reglamento General de Extensión Universitaria, vigente desde el año 2005. En esta disposición interna se enuncian una diversidad de actividades llevadas a cabo en las Facultades e Institutos que integran la gran familia de esta centenaria universidad.

Tanto el Plan Estratégico como el Reglamento General de Extensión Universitaria, permitieron a la UNA, en estos últimos años, potenciar significativamente las actividades de extensión en todo el cuerpo de la universidad, haciendo posible un contacto directo de estudiantes y profesores con la comunidad circundante, facilitando las alianzas y cooperaciones no sólo con municipios y empresas, sino también con organismos y entidades públicas y privadas, nacionales e internacionales, así como con organizaciones de la sociedad civil, que en conjunto permiten enriquecer el concepto de extensión universitaria y el papel 
que a ésta le cabe para transferir conocimiento y tecnología a la sociedad. Estas actividades son llevadas a cabo por las 11 unidades académicas que abarcan desde las Ciencias Agrarias, Arquitectura, Ingeniería, hasta las de Ciencias Sociales como las facultades de Trabajo Social, Ciencias Económicas, entre otras.

La FCE, como unidad componente de la UNA, apunta a contribuir, con su quehacer y modalidades propias, a la preparación de ciudadanos con las competencias necesarias para insertarse en los diferentes sectores promotores del desarrollo de la sociedad paraguaya, y ha articulado la modalidad de la extensión universitaria para lograrlo.

Bajo las orientaciones de la disposición institucional de la UNA como lo es el Reglamento General de Extensión Universitaria, la FCE-UNA aprobó la Resolución $N^{\circ} 1$ en el Acta $N^{\circ} 15$ del 11 de agosto del año 2008 por la que se establece el Reglamento Interno de Extensión Universitaria de la FCE. Así, la FCE, a través de la DES, inició el proceso de implementación de varios proyectos de extensión universitaria como los cursos extracurriculares, los programas de capacitación a la comunidad, las charlas educativas de interés social, e incluso campañas de concienciación sobre higiene bucal, prevención de enfermedades contagiosas y cuidado del medio ambiente.

Cabe destacar que, en el mes de setiembre del año 2015, la comunidad educativa de la UNA vivencia un acontecimiento histórico denominado Primavera Estudiantil ${ }^{2}$ que clamaba a la academia que se adapte a los cambios requeridos por la globalización; a partir de entonces, se inicia el proceso de modificaciones en el estatuto y se apunta a potenciar el desarrollo de acciones que conduzcan a cumplir con los fines principales de la docencia, la investigación y la extensión universitaria.

En las unidades académicas de la UNA se reorientan las actividades de extensión y se diseñan bajo el contexto emergente de que la sociedad respalde y confíe nuevamente en la institución de educación superior. Así, la FCE-UNA, por Resolución N 11 del Acta Nº del 27 de marzo de 2017 del Consejo Directivo de la Facultad de Ciencias Económicas, procedió a actualizar la Escala de Ponderación para las Actividades de Extensión Universitaria y se abocó, a través de la Dirección de Extensión y Servicios, a encarar proyectos de vinculación social.

La Dirección de Extensión y Servicios de la FCE-UNA, comenzó a trabajar en programas de asistencia a sectores vulnerables, como los pequeños productores agroganaderos, microempresarios, asentamientos urbanos, entre otros; ubicados dentro del radio de acción de la sede central y filiales, localizados en los departamentos de Central, Cordillera, Paraguarí, Caaguazú, San Pedro y Presidente Hayes de Paraguay; lo que dio lugar a la construcción de la escala de valoración de proyectos de extensión universitaria mediante la Resolución N 4 del Acta N 9 del 16 de mayo de 2018 del Consejo Directivo de la Facultad de Ciencias Económicas.

En dicha escala de valoración de las actividades de extensión universitaria, cuentan con más horas de créditos aquellas relacionadas con el desarrollo socio-comunitario con enfoque en la ciencia, la tecnología

2 Primavera Estudiantil: Movimiento organizado por los estudiantes de las diversas unidades académicas, denominado \#UNANOTECALLES, con el objetivo de iniciar la reforma democrática en los estatutos de la Universidad Nacional de Asunción (UNA). 
y el humanismo y los servicios técnicos profesionales, de manera a incentivar la realización de estas iniciativas de vinculación social.

Con esta disposición emanada de la FCE-UNA, la Dirección de Extensión y Servicios puso en marca el diseño de proyectos enmarcados en las líneas de acción enfocados al desarrollo socio-comunitario, a la producción de materiales educativos, didácticos y difusión, a programa educativo, social, cultural y recreativo y a espacios de intercambio de saberes.

\section{La vinculación de la FCE-UNA con sectores vulnerables a través de la extensión universitaria}

Como se ha señalado anteriormente, la vinculación de Universidad, Sociedad y Estado, implica atender, desde la Academia, a aquellos sectores de la población que no cuentan con posibilidades de acceso no solo a los servicios básicos sino incluso a alternativas de mejoramiento socio económico. Estos sectores presentan condiciones de vulnerabilidad, entre los cuales pueden citarse a grupos poblacionales como las niñas, los niños y jóvenes en situación de calle, los migrantes, las personas con discapacidad, los adultos mayores y la población indígena, los micro emprendedores productivos, entre otros. Atendiendo que dicha población enfrenta un conjunto de situaciones que las excluyen de posibles beneficios y acceso a servicios como ser, salud, educación, trabajo digno, y el ejercicio de derechos básicos fundamentales para así, lograr la inserción activa de los ciudadanos y ciudadanas a la vida política y socioeconómica del país; por ello precisan de la asistencia de la Academia para poder desarrollar y potenciar los escasos recursos con los que cuentan.

En este contexto, como lo reseñan Alvarez \& Fonseca (2018) tanto en el Plan Estratégico como en el Reglamento General de Extensión Universitaria en la UNA, se han resaltado y potenciado significativamente a las actividades de extensión en todo el cuerpo de la universidad, a fin de que las mismas posibiliten un contacto directo de estudiantes y profesores con la comunidad circundante. Esto solo se puede lograr con el trabajo en equipo, por lo cual en los citados documentos, se han delineado mecanismos que faciliten las alianzas y cooperaciones, no sólo con municipios y empresas, sino también con organismos y entidades públicas y privadas, nacionales e internacionales, así como con organizaciones de la sociedad civil, que en conjunto, coadyuvan al enriquecimiento del concepto de extensión universitaria y el papel que a ésta le cabe para transferir conocimiento y tecnología a la sociedad.

En este contexto de lo señalado por los autores mencionados, la FCE en mayo del 2017, creó el Consultorio Universitario Contable, Administrativo y Económico (CUCAE) a disposición de micro emprendedores, dueños de negocios, personas en situación de autoempleo y empleados sin conocimientos de contabilidad, administración y economía.

El CUCAE brinda asistencia técnica, profesional y académica en las áreas de Contaduría Pública, Administración y Economía como parte principal de su programa de extensión universitaria, aplicando conocimientos científicos y técnicos de las distintas disciplinas de la formación profesional a microempresarios, dueños de negocios, empleados, gobernaciones departamentales, municipalidades del interior, líderes comunitarios y público en general. 
Inicialmente, la Dirección de Extensión y Servicios de la FCE, a través del CUCAE, entre los meses de junio y julio del año 2017, inició los primeros acercamientos con los sectores vulnerables ubicados en el mercado municipal de la ciudad de San Lorenzo, departamento Central, donde a los micro emprendedores se les instruyó en la elaboración del presupuesto de caja como instrumento financiero para realizar planes de negocio.

Posteriormente, entre agosto y setiembre del año 2017, se asistieron a los artesanos zapateros residentes en la ciudad Pirayú y a los oleros de la ciudad de Tobatí del departamento de Cordillera y a las artesanas del poyvi ${ }^{3}$ ubicadas en la comunidad de Espartillar en el departamento de Carapeguá.

A través del CUCAE se realizaron las actividades de asistencia que incluyeron la auditoría de la gestión administrativa y asesoría en estructura de costos a micro-empresarios y artesanos; asesoramiento profesional para formalización de organizaciones y emprendimientos y educación financiera.

Desde entonces, el CUCAE se constituye en una valiosa articulación para diseñar y desarrollar programas de extensión universitaria que fortalezcan el vínculo entre la facultad y la sociedad y propone actividades de interacción universidad-sociedad en la que los estudiantes puedan dar su contribución a la solución de las diversas problemáticas de nuestras sociedades son su centro de acción.

En marzo del año 2019 la FCE, a través de la Dirección de Extensión y Servicios, realizó un convenio interinstitucional con la Sub-Secretaría de Estado de Tributación (SET) del Ministerio de Hacienda para la instalación del Núcleo de Asistencia Tributaria (NAT), programa de la Euro Social + que apunta a desarrollar y fortalecer la cultura tributaria y la conciencia social ciudadana. Desde el NAT se brindan orientaciones básicas sobre el Registro Único del Contribuyente (RUC), el Sistema de Facturación Electrónica (SIFEN), los alcances de los impuestos vigentes y todas las herramientas brindadas por la Sub Secretaría de Estado de Tributación (SET) del Ministerio de Hacienda.

Este cúmulo de experiencias obtenidas en la vinculación de la población y la academia fue muy positivo, lo que motivó a la FCE a ampliar su radio de acción y a replicar las actividades, durante los años 2018 y 2019, a más comunidades.

Desde dichos años, la Dirección de Extensión y Servicios de la FCE-UNA ha propiciado actividades de extensión dentro de la línea de acción denominada de desarrollo comunitario, cambiando la mentalidad o el enfoque de las actividades de extensión tradicionalmente llevadas a cabo, puesto que, el nuevo enfoque incluye brindar no solo capacitaciones en diversos temas, sino también apoyar con asistencia técnica a pobladores de las comunidades o asentamientos, a quienes se ha instruido en temas como educación financiera familiar, presupuesto de gastos y asociatividad. En este contexto, otro tipo de actividades de extensión llevadas a cabo en poblaciones vulnerables se ha concretado en una comunidad caracterizada como artesanos del calzado, a quienes se ha capacitado para que puedan identificar su costo de producción, contribuyendo a desarrollas dicha habilidad a partir de la cual puedan cuenten con conocimientos para calcular un parámetro de fijación del precio final de sus productos. Asimismo, en otra

3 Poyvi: tejido artesanal de Paraguay confeccionado a partir de materiales reciclados de tela y/o reciclables como la arpillera, hilos de algodón e hilos de lanilla. 
comunidad caracterizada como de artesanas del ñandutí ${ }^{4}$ y hamacas de hilo, artesanas del poyvi, poncho y artesanos de pelotas de cuero, se les capacita en educación financiera y se realiza una fuerte promoción de marketing realizando la Expo Eco MIPYMES en la que participan para vender sin intermediaciones sus productos. Otra comunidad que ha sido asistida está compuesta por artesanos oleros, a quienes se les ha capacitado para formar asociaciones y así fortalecer el gremio de productores; unido a la capacitación no solo para identificar su costo de producción sino también canales alternativos de venta. Además, se les ha brindado cursos de educación financiera y asesoramiento profesional con respecto a su nivel de endeudamiento. También, se ha desarrollado en la comunidad de los fabricantes de dulces artesanales, cursos de capacitación sobre educación financiera, y se ha brindado asesoramiento sobre el tema de costo y asesoramiento profesional en cadenas de comercialización.

\section{La extensión universitaria en épocas de COVID 19 y el cambio de mentalidad para un nuevo enfoque de las actividades extensionistas}

El ser humano es capaz de afrontar situaciones extremas, y superarlas, aunque ello depende mucho de lo aprendido, aprehendido y convivido. En este sentido, un término que se ajusta a la realidad surgida con esta Pandemia del 2020 es el de la resiliencia, que, si bien tiene varias conceptualizaciones dadas por muchos especialistas que han estudiado el término; en el ámbito educativo, y en el contexto del tema tratado en este artículo, se asume que la resiliencia es la capacidad o habilidad de todo ser humano, que ha enfrentado una situación adversa, para superar y resurgir, aprendiendo de la situación que enfrenta y adaptándose a los cambios que surjan para construir una vida significativa y productiva a partir de la misma.

Teniendo en cuenta lo anterior, corresponde a la Academia, ámbito en el cual se forman a profesionales para el presente y futuro, ser lo más resiliente posible, con el objetivo principal que los estudiantes y egresados puedan construir y crecer como personas integras, e incluso los docentes, requieren por ello desarrollar resiliencia, como lo expresan Noriega, et al (2015).

Por ello, Noriega, et al (2015) también puntualiza que la educación en el siglo XXI está en la búsqueda de medios para contribuir a la calidad de vida en la sociedad, por lo cual precisa dar está prioridad a dos pilares importantes de la educación como son: aprender a aprender y aprender a vivir juntos; dado que los autores consideran que dichos pilares son los cimientos para el cambio educativo.

La magnitud y la gravedad de la Pandemia del COVID-19 que continúa azotando al mundo, claramente ascendieron al nivel de una amenaza a la salud pública en Paraguay que justificó restricciones a ciertos derechos e impuso la cuarentena y el aislamiento, que lógicamente limitan la libertad de movimiento para la extensión universitaria de la FCE-UNA. Sin embargo, la explosión del virus COVID19 ha puesto de relieve la intervención positiva que la universidad puede generar y en la FCE-UNA, luego de analizar en equipo la situación enfrentada en el año 2020, ha considerado necesario el cambio de mentalidad para

4 Ñanduti (tela de araña en castellano), poyvi (tejido en castellano), constituyen artesanía típica de Paraguay, utilizando denominación en lengua guaraní. Nanduti es un encaje de agujas que se teje sobre bastidores en círculos radiales, con bordados de motivos geométricos o zoomorfos (formas de animales). Poyvi, se utiliza como tejido para mantas, cobertores, ponchos, alfombras y hasta tapices. 
implementar las actividades de extensión universitaria, y posibilitar el mantenimiento de los programas desarrollados en los años 2018-2019. Esto ha implicado que la FCE-UNA trabaje articuladamente para diseñar el sendero tecnológico que permita mantener el vínculo con las comunidades con las cuales ha llevado a cabo el abordaje comunitario, más aún, considerando que las mismas pertenecen a sectores vulnerables, que requieren acompañamiento y ayuda mutua.

Es así que, los abordajes comunitarios realizados in situ en el último bimestre del año 2019 han permitido identificar la necesidad de dar continuidad en el año 2020 a las actividades de extensión universitaria llevadas a cabo con los artesanos de diversos rubros, donde el denominador común fue brindar capacitación y asistencia técnica a los mismos.

La FCE-UNA para ejecutar acciones sostenibles y permanentes que aseguren que la relación no se sustente en un extractivismo meramente académico, ha adoptado, a través de la Dirección de Extensión y Servicios, en el año 2020, la implementación del uso de herramientas virtuales en las actividades de extensión universitaria, utilizando las herramientas de las Tecnologías de Información y Comunicación TIC, a fin de desarrollar las actividades intramuros que apunten a contribuir a la formación integral y multidisciplinaria de la comunidad universitaria, y que además posibiliten contemplar los canales para ejecutar actividades y grilla de acción extramuros que viabilicen la continuidad del acceso a servicios profesionales y técnicos puestos a disposición de la población.

Uno de los medios utilizados para implementar lo señalado anteriormente es que la Dirección de Extensión y Servicios de la FCE-NA, a través del CUCAE, ha implementado la virtualización de los servicios del Núcleo de Asistencia Tributaria (NAT); y para ello, se encuentra utilizando la herramienta Microsoft Teams y el Google Meet, con sus múltiples aplicaciones que posibilitan la realización de grupos de chat, reuniones, llamadas y colaboración del equipo de estudiantes consultores con la población en forma virtual. Estas herramientas virtuales son utilizadas por docentes y estudiantes no solo en la planificación de las actividades de extensión universitaria, para el abordaje comunitario, implementando la aplicación de cuestionarios virtuales para diagnóstico y caracterización de las comunidades; sino también, como medio para elaborar los informes, análisis y estudios, productos del procesamiento de los datos e informaciones obtenidas con dichas herramientas.

Igualmente, ha desarrollado la asistencia virtual a través del Núcleo de Asistencia Empresarial (NAE), programa que se encuentra desarrollado en convenio con el Ministerio de Industria y Comercio (MIC), a través del cual se ha brindado asistencia a las micro y pequeñas empresas en el Sistema Unificado de Apertura y Cierre de Empresas (SUACE) y la Ventanilla Única de Exportación (VUE), así como se ha ofrecido diversos servicios y productos que apuntan a fortalecer a los emprendedores de Paraguay, como los relativos a asesoramiento para el manejo administrativo, estructura de costos de producción, canales de obtención de créditos y educación financiera.

En el año 2020 con el proyecto de extensión denominado Mentoring inició con un abordaje comunitario donde se detectaron 99 (noventa y nueve) micro empresas artesanales cuya actividad se constituye en el único medio de generación de ingresos para el núcleo familiar que aglutina. Posteriormente, se evidenció las áreas de requerimiento de asistencia técnica que asegurara la sostenibilidad y sustentabilidad de la 
idea de negocio en el tiempo. Los docentes tutores trabajaron en el programa para la asistencia técnica personalizada de cada micro empresa en la que los estudiantes de las carreras de Contaduría Pública, Administración y Economía fungieron de Mentoring empresarial a lo largo de un semestre en el marco de la extensión universitaria utilizando la tecnología como aliado ya que la vinculación se realizó mediante el Google Meet, el Zoom, la video llamada de WhatsApp y el Google form.

\section{Lecciones aprendidas y compartidas sobre el nuevo enfoque de la extensión universitaria en épocas de COVID 19}

De todo lo expuesto con respecto a lo realizado en la adecuación hacia un nuevo enfoque para realizar actividades de extensión universitaria en este año 2020, enfrentando la Pandemia y reorientando la educación, se comparten las siguientes lecciones aprendidas:

La extensión implica un aprendizaje recíproco, donde el centro neurálgico de la actividad es el estudiante para que pueda conocer la realidad de su profesión, enriquecerla y redimensionar toda su actividad académica.

La UNA es una organización que funciona en su sociedad y genera impactos tanto sobre la comunidad educativa como sobre su entorno social y natural lo que la compromete a responder de modo ético e inteligente por estos impactos para que sean, en la medida de lo posible, positivos y no negativos.

La FCE asumió el desafío de la vinculación social a través de la extensión universitaria y apuesta al desarrollo de servicios comunitarios donde se propicia un espacio ideal para que se compartan conocimientos científicos, tecnológicos y culturales para resolver alguna necesidad de una comunidad determinada y dar respuestas que apuntan al desarrollo de dicha comunidad. Por tanto, en el corto plazo, deberá prever mecanismos para articular que necesariamente todas las actividades que se deban ejecutar, partan de la identificación de las necesidades prioritarias de toda la sociedad para que sean atendidos por los proyectos de vinculación.

A través de la implementación de los diversos programas de extensión universitaria con abordaje comunitario, la FCE-UNA ha actuado en forma resiliente, adecuando su forma de llegar a las comunidades localizadas en su área de influencia, contribuyendo no solo a formar integralmente a los estudiantes sino también a incidir en la población más vulnerable, capacitándola y asistiéndola en sus necesidades, principalmente, las que le competen y que se encuentran vinculadas a la generación de ingresos, ya sean familiares o microempresariales.

En este cambio de mentalidad, y considerando que el logro de los objetivos académicos solo es posible con el esfuerzo del trabajo en equipo, la FCE-UNA ha construido acuerdos, fortalecido alianzas e implementado convenios, con entidades, organizaciones e instituciones, de todos los sectores de la sociedad paraguaya, tanto público, privado como de la economía social.

Es importante destacar que, la situación mundial vivida en este año 2020, exige de todos, y principalmente de las instituciones educativas, el desarrollo de la capacidad para sobreponerse a esta situación crítica, a 
fin de adaptarse con nuevas iniciativas que posibiliten la formación de nuevos ciudadanos centrados en valores y principios, que conozcan su realidad y los medios o recursos para transformarla. Para lograrlo, un instrumento importante es desarrollar actividades de extensión universitaria, contando con los recursos, no solo humanos sino tecnológicos, puestos al servicio de la educación para una formación integral de los futuros profesionales.

\section{REFERENCIAS}

Alvarez, L., \& Fonseca, S. (2018). Vinculación de la Facultad de Ciencias Económicas de la Universidad Nacional de Asunción con la sociedad mediante la extensión universitaria. Población y Desarrollo. $N^{\circ} 46,83-92$.

Chuaqui, B. (2002). Acerca de la historia de las universidades. Revista chilena de pediatría, v. 73 , n. 6. Santiago, nov. 2002, paginas 563-565. Disponible en: https://www.scielo.cl/scielo.php?script=sci_ arttext\&pid =S0370-41062002000600001

Del Huerto, M. H. (2006). La extensión universitaria como vía para fortalecer los vínculos universidadsociedad desde la promoción de la salud. Revista Cubana de Salud Pública. La Habana. Volumen $33,33-39$.

Di Mari, D. (2013). La importancia de la extensión universitaria. Buenos Aires: Anuario Escuela de ARchivología IV 2012-2013 Universidad Nacional de Córdoba.

Facultad de Ciencias Económicas (2008). Acta No. 15 (C.D. No.15 11/08/2008). Resolución N¹ "REGLAMENTO INTERNO DE EXTENSIÓN UNIVERSITARIA DE LA FACULTAD DE CIENCIAS ECONÓMICAS DE LA UNIVERSIDAD NACIONAL DE ASUNCIÓN". Recuperado de: https://www. eco.una.py/application/files/6415/2155/7632/Reglamento_interno_extension_2008.pdf

Facultad de Ciencias Económicas (2018). Acta No. 09 (C.D. No.09 16/05/2018). Resolución N4“ESCALA DE VALORACIÓN DE PROYECTOS DE EXTENSIÓN UNIVERSITARIA DE LA FACULTAD DE CIENCIAS ECONÓMICAS DE LA UNIVERSIDAD NACIONAL DE ASUNCIÓN". https://www.eco.una.py/ application/files/6115/6113/9452/ESCALA_DE_VALORACION.pdf

Honorable Cámara de Representantes (1956). Ley No 356 "QUE ESTABLECE LA CARTA ORGÁNICA DE LA UNIVERSIDAD NACIONAL DE ASUNCIÓN". Recuperado de: http://www.bacn.gov.py/leyesparaguayas/2613/establece-la-carta-organica-de-la-universidad-nacional-de-asuncion

Noriega Aguilar, G.; Angulo, B.; Angulo, G. (2015) La resiliencia en la educación, la escuela y la vida Octubre de 2015. Disponible en: https://dialnet.unirioja.es/descarga/articulo/6349252.pdf

Poder Legislativo (2013). Ley No 4995De Educación Superior. Asunción, 2 de agosto de 2013. Recuperado de: http://www.cones.gov.py/ley-4995-de-educacion-superior/

Universidad Nacional de Asunción (2005). Acta No. 22 (A.S. No. 22/02/2005) Resolución No. 428-002005 "POR LA CUAL SE APRUEBA EL REGLAMENTO GENERAL DE EXTENSION UNIVERSITARIA". Recuperado de: https://www.una.py/images/stories/Universidad/DisposicionesLegales/428_2005. pdf

Tomassino, H., \& Cano, A. (2016). Modelos de extensión universitaria en las universidades latinoamericanas en el Siglo XXI: tendencias y controversias. Universidades $N^{\circ} 67,7-24$.

Universidad Nacional de Asunción (2017). Estatuto Aprobado, sancionado y puesto en vigencia por la Asamblea Universitaria el 5 de diciembre de 2017. Asunción-Paraguay. Recuperado de: https:// www.una.py/wp-content/uploads/2019/07/Estatuto_de_la_UNA_2017.pdf 